\title{
Soft Bitopological Spaces
}

\author{
Basavaraj M. Ittanagi \\ Department of Mathematics, \\ Siddaganga Institute of Technology, \\ Tumkur-572103, Karnataka State, India
}

\begin{abstract}
In this paper, introduce and study the concept of soft bitopological spaces which are defined over an initial universe with a fixed set of parameters. Also introduce and investigate some new separation axioms called pairwise soft $T_{0}$, pairwise soft $T_{1}$ and pairwise soft $T_{2}$ spaces and study some of their basic properties in soft bitopological spaces.
\end{abstract}

\section{General Terms:}

2010 Mathematics Subject Classification: 54E55, 54D10.

\section{Keywords:}

Soft sets, Soft topology, Soft bitopology.

\section{INTRODUCTION}

In the year 1999, Russian researcher Molodtsov[6], initiated the concept of soft sets as a new mathematical tool to deal with uncertainties while modeling problems in engineering physics, computer science, economics, social sciences and medical sciences. In 2003, Maji, Biswas and Roy[5], studied the theory of soft sets initiated by Molodtsov. They defined equality of two soft sets, subset and super set of a soft set, complement of a soft set, null soft set and absolute soft set with examples. Soft binary operations like AND, OR and also the operations of union and intersection were also defined. In 2005, D. Chen[2], presented a new definition of soft set parametrization reduction and a comparison of it with attribute reduction in rough set theory.

Topological structures of soft set have been studied by some authors in recent years. In 2011, M. Shabir et al. and Naim Cagman et al. initiated the study of soft topology and soft topological spaces independently. M. Shabir and M. Naz[7], introduced the notion of soft topological spaces which are defined over an initial universe with a fixed set of parameters and showed that a soft topological space gives a parameterized family of topological spaces. They introduced the definitions of soft open sets, soft closed sets, soft interior, soft closure and soft separation axioms. Also they obtained some interesting results for soft separation axioms which are really valuable for research in this field. N. Cagman, S. Karatas and S. Enginoglu[1], defined the soft topology on a soft set and presented its related properties and foundations of the theory of soft topological spaces.
In 1963, J. C. Kelly[4], first initiated the concept of bitopological spaces. He defined a bitopological space $\left(X, \tau_{1}, \tau_{2}\right)$ to be a set $X$ equipped with two topologies $\tau_{1}$ and $\tau_{2}$ on $X$ and initiated the systematic study of bitopological space. Later work done by C. W. Patty[8], I. L. Reilly[9] and others. Reilly discussed separation axioms properties in bitopological spaces.

The following definitions which are prerequisites for present study.

1.1 Definition[6]: Let $U$ be an initial universe and $E$ be a set of parameters. Let $P(U)$ denote the power set of $U$ and $A$ be a nonempty subset of $E$. A pair $(F, A)$ is called a soft set over $U$, where $F$ is a mapping given by $F: A \rightarrow P(U)$.

In other words, a soft set over $U$ is a parametrized family of subsets of the universe $U$. For $\epsilon \in A, F(\epsilon)$ may be considered as the set of $\epsilon$-approximate elements of the soft set $(F, A)$. Clearly, a soft set is not a set.

1.2 Definition[5]: The complement of a soft set $(F, A)$ is denoted by $(F, A)^{c}$ and is defined by $(F, A)^{c}=\left(F^{c}, \neg A\right)$ where $F^{c}$ : $\neg A \rightarrow P(U)$ is a mapping given by $F^{c}(\alpha)=U-F(\alpha)$ for all $\alpha \in \neg A$.

Let us call $F^{c}$ to be the soft complement function of $F$. Clearly $\left(F^{c}\right)^{c}$ is the same as $F$ and $\left((F, A)^{c}\right)^{c}=(F, A)$.

1.3 Definition[5]: A soft set $(F, A)$ over $U$ is said to be a $N U L L$ soft set denoted by $\phi$ if for all $\epsilon \in A, F(\epsilon)=\phi$ (null set).

1.4 Definition[5]: The union of two soft sets of $(F, A)$ and $(G, B)$ over the common universe $U$ is the soft set $(H, C)$, where $C=$ $A \cup B$ and for all $e \in E$,

$$
H(e)= \begin{cases}F(e) & \text { ife } \in A-B \\ G(e) & \text { ife } \in B-A \\ F(e) \cup G(e) & \text { ife } \in A \cap B\end{cases}
$$

and is written as $(F, A) \cup(G, B)=(H, C)$

1.5 Definition[3]: The intersection $(H, C)$ of two soft sets $(F, A)$ and $(G, B)$ over a common universe $U$, denoted $(F, A) \cap(G, B)$, is defined as $C=A \cap B$, and $H(e)=F(e) \cap G(e)$ for all $e \in C$.

1.6 Definition[7]: Let $(F, E)$ be a soft set over $X$ and $x \in X$. We say that $x \in(F, E)$ read as $x$ belongs to the soft set $(F, E)$ whenever $x \in F(\alpha)$ for all $\alpha \in E$. 
Note that for any $x \in X, x \notin(F, E)$ if $x \notin F(\alpha)$ for some $\alpha \in E$.

1.7 Definition[7]: Let $Y$ be a non-empty subset of $X$, then $Y$ denotes the soft set $(Y, E)$ over $X$ for which $Y(\alpha)=Y$, for all $\alpha \in E$.

1.8 Definition[7]: Let $x \in X$, then $(x, E)$ denotes the soft set over $X$ for which $x(\alpha)=\{x\}$, for all $\alpha \in E$.

1.9 Definition[7]: Let $(F, E)$ be a soft set over $X$ and $Y$ be a nonempty subset of $X$. Then the sub soft set of $(F, E)$ over $Y$ denoted by $\left({ }^{Y} F, E\right)$, is defined as follows

${ }^{Y} F(\alpha)=Y \cap F(\alpha)$, for all $\alpha \in E$. In other words $\left({ }^{Y} F, E\right)=$ $Y \cap(F, E)$.

1.10 Definition[7]: The relative complement of a soft set $(F, A)$ is denoted by $(F, A)^{c}$ and is defined by $(F, A)^{c}=\left(F^{c}, A\right)$ where $F^{c}: A \rightarrow P(U)$ is a mapping gives by $F^{c}(\alpha)=U-F(\alpha)$ for all $\alpha \in A$.

1.11 Definition[7]: Let $\tau$ be the collection of soft sets over $X$, then $\tau$ is said to be a soft topology on $X$, if

(1) $\phi, X$ belong to $\tau$

(2) the union of any number of soft sets in $\tau$ belongs to $\tau$

(3) the intersection of any two soft sets in $\tau$ belongs to $\tau$.

The triplet $(X, \tau, E)$ is called a soft topological space over $X$.

1.12 Definition[7]: Let $(X, \tau, E)$ be a soft topological space over $X$, then the members of $\tau$ are said to be soft open sets in $X$.

1.13 Definition[7]: Let $(X, \tau, E)$ be a soft topological space over $X$. A soft open set $(F, E)$ over $X$ is said to be a soft closed set in $X$, if its relative complement $(F, E)^{c}$ belongs to $\tau$.

1.14 Definition[7]: Let $(X, \tau, E)$ be a soft topological space over $X$ and $Y$ be a non-empty subset of $X$. Then $\tau_{Y}=\left\{\left({ }^{Y} F, E\right)\right.$ : $(F, E) \in \tau\}$ is said to be the soft relative topology on $Y$ and $\left(Y, \tau_{Y}, E\right)$ is called a soft subspace of $(X, \tau, E)$.

1.15 Definition[7]: Let $(X, \tau, E)$ be a soft topological space over $X$ and $x, y \in X$ such that $x \neq y$.

i) If there exist soft open sets $(F, E)$ and $(G, E)$ such that $x \in$ $(F, E)$ and $y \notin(F, E)$ or $y \in(G, E)$ and $x \notin(G, E)$, then $(X, \tau, E)$ is called a soft $T_{0}$ space.

ii) If there exist soft open sets $(F, E)$ and $(G, E)$ such that $x \in$ $(F, E)$ and $y \notin(F, E)$ and $y \in(G, E)$ and $x \notin(G, E)$, then $(X, \tau, E)$ is called a soft $T_{1}$ space.

iii) If there exist soft open sets $(F, E)$ and $(G, E)$ such that $x \in$ $(F, E), y \in(G, E)$ and $(F, E) \cap(G, E)=\phi$, then $(X, \tau, E)$ is called a soft $T_{2}$ space.

1.16 Definition[4]: Let $X$ be a non-empty set and $\tau_{1}$ and $\tau_{2}$ be two different topologies on $X$. Then $\left(X, \tau_{1}, \tau_{2}\right)$ is called a bitopological space.

\section{SOFT BITOPOLOGICAL SPACES}

Let $X$ be an initial universe set and $E$ be the non-empty set of parameters.

2.1 Definition: Let $\left(X, \tau_{1}, E\right)$ and $\left(X, \tau_{2}, E\right)$ be the two different soft topologies on $X$. Then $\left(X, \tau_{1}, \tau_{2}, E\right)$ is called a soft bitopological space.
The two soft topologies $\left(X, \tau_{1}, E\right)$ and $\left(X, \tau_{2}, E\right)$ are independently satisfy the axioms of soft topology. The members of $\tau_{1}$ are called $\tau_{1}$ soft open sets and the complements of $\tau_{1}$ soft open sets are called $\tau_{1}$ soft closed sets.

Similarly, The members of $\tau_{2}$ are called $\tau_{2}$ soft open sets and the complements of $\tau_{2}$ soft open sets are called $\tau_{2}$ soft closed sets.

Throughout this paper $\left(X, \tau_{1}, \tau_{2}, E\right)$ denote soft bitopological space over $X$ on which no separation axioms are assumed unless explicitly stated.

2.2 Example: Let $X=\left\{h_{1}, h_{2}, h_{3}\right\}, E=\left\{e_{1}, e_{2}\right\}$ and $\tau_{1}=\left\{\phi, X,\left(F_{1}, E\right),\left(F_{2}, E\right),\left(F_{3}, E\right),\left(F_{4}, E\right),\left(F_{5}, E\right)\right\}$ and $\tau_{2}=\left\{\phi, X,\left(G_{1}, E\right),\left(G_{2}, E\right),\left(G_{3}, E\right),\left(G_{4}, E\right),\left(G_{5}, E\right),\left(G_{6}, E\right)\right.$, $\left.\left(G_{7}, E\right)\right\}$, where $\left(F_{1}, E\right),\left(F_{2}, E\right),\left(F_{3}, E\right),\left(F_{4}, E\right),\left(F_{5}, E\right)$, $\left(G_{1}, E\right),\left(G_{2}, E\right),\left(G_{3}, E\right),\left(G_{4}, E\right),\left(G_{5}, E\right),\left(G_{6}, E\right),\left(G_{7}, E\right)$ are soft sets over $X$, defined as follows

$F_{1}\left(e_{1}\right)=\left\{h_{2}\right\} F_{1}\left(e_{2}\right)=\left\{h_{1}\right\}$

$F_{2}\left(e_{1}\right)=\left\{h_{2}, h_{3}\right\} F_{2}\left(e_{2}\right)=\left\{h_{1}, h_{2}\right\}$

$F_{3}\left(e_{1}\right)=\left\{h_{1}, h_{2}\right\} F_{3}\left(e_{2}\right)=X$

$F_{4}\left(e_{1}\right)=\left\{h_{1}, h_{2}\right\} F_{4}\left(e_{2}\right)=\left\{h_{1}, h_{3}\right\}$

$F_{5}\left(e_{1}\right)=\left\{h_{2}\right\} F_{5}\left(e_{2}\right)=\left\{h_{1}, h_{2}\right\}$

$G_{1}\left(e_{1}\right)=\left\{h_{1}, h_{2}\right\} G_{1}\left(e_{2}\right)=\left\{h_{1}, h_{2}\right\}$

$G_{2}\left(e_{1}\right)=\left\{h_{2}\right\} G_{2}\left(e_{2}\right)=\left\{h_{1}, h_{3}\right\}$

$G_{3}\left(e_{1}\right)=\left\{h_{2}, h_{3}\right\} G_{3}\left(e_{2}\right)=\left\{h_{1}\right\}$

$G_{4}\left(e_{1}\right)=\left\{h_{2}\right\} G_{4}\left(e_{2}\right)=\left\{h_{1}\right\}$

$G_{5}\left(e_{1}\right)=\left\{h_{1}, h_{2}\right\} G_{5}\left(e_{2}\right)=X$

$G_{6}\left(e_{1}\right)=X \quad G_{6}\left(e_{2}\right)=\left\{h_{1}, h_{2}\right\}$

$G_{7}\left(e_{1}\right)=\left\{h_{2}, h_{3}\right\} G_{7}\left(e_{2}\right)=\left\{h_{1}, h_{3}\right\}$

Then $\left(X, \tau_{1}, \tau_{2}, E\right)$, is a soft bitopological space.

2.3 Example: Let $X$ be an initial universe set and $E$ be the nonempty set of parameters. Soft indiscrete topology $\tau_{1}=\{\phi, X\}$ and soft discrete topology $\tau_{2}$ is the collection of all soft sets defined over $X$. Then $\left(X, \tau_{1}, \tau_{2}, E\right)$ is a soft bitopological space.

2.4 Definition: Let $\left(X, \tau_{1}, \tau_{2}, E\right)$ be a soft bitopological space over $X$ and $Y$ be a non-empty subset of $X$. Then $\tau_{1 Y}=\left\{\left({ }^{Y} F, E\right)\right.$ : $\left.(F, E) \in \tau_{1}\right\}$ and $\tau_{2 Y}=\left\{\left({ }^{Y} G, E\right):(G, E) \in \tau_{2}\right\}$ are said to be the relative topologies on $Y$. Then $\left\{Y, \tau_{1 Y}, \tau_{2 Y}, E\right\}$ is colled a relative soft bitopological space of $\left(X, \tau_{1}, \tau_{2}, E\right)$.

2.5 Theorem: If $\left(X, \tau_{1}, \tau_{2}, E\right)$ is a soft bitopological space then $\tau=\tau_{1} \cap \tau_{2}$ is a soft topological space over $X$.

Proof: 1$)$. $\phi, X$ belong to $\tau_{1} \cap \tau_{2}=\tau$

2). Let $\left\{\left(F_{i}, E\right): i \in I\right\}$ be a family of soft sets in $\tau_{1} \cap \tau_{2}=\tau$.

Then $\left\{\left(F_{i}, E\right) \in \tau_{1}\right\}$ and $\left\{\left(F_{i}, E\right) \in \tau_{2}\right\}$ for all $i \in I$.

Therefore $\bigcup_{i \in I}\left(F_{i}, E\right) \in \tau_{1}$ and $\bigcup_{i \in I}\left(F_{i}, E\right) \in \tau_{2}$.

Thus $\bigcup_{i \in I}\left(F_{i}, E\right) \in \tau_{1} \cap \tau_{2}=\tau$.

$3)$. Let $(F, E),(G, E) \in \tau_{1} \cap \tau_{2}=\tau$.

Then $(F, E),(G, E) \in \tau_{1}$ and $(F, E),(G, E) \in \tau_{2}$.

Since $(F, E) \cap(G, E) \in \tau_{1}$ and $(F, E) \cap(G, E) \in \tau_{2}$.

Therefore $(F, E) \cap(G, E) \in \tau_{1} \cap \tau_{2}=\tau$.

Thus $\tau_{1} \cap \tau_{2}=\tau$ defines a soft topology on $X$.

2.6 Remark: If $\left(X, \tau_{1}, \tau_{2}, E\right)$ is a soft bitopological space then $\tau_{1} \cup \tau_{2}$ is not a soft topological space over $X$.

2.7 Example: Let $X=\left\{h_{1}, h_{2}, h_{3}\right\}, E=\left\{e_{1}, e_{2}\right\}$ and $\tau_{1}=\left\{\phi, X,\left(F_{1}, E\right),\left(F_{2}, E\right),\left(F_{3}, E\right),\left(F_{4}, E\right)\right\}$ and $\tau_{2}=\left\{\phi, X,\left(G_{1}, E\right),\left(G_{2}, E\right),\left(G_{3}, E\right),\left(G_{4}, E\right),\left(G_{5}, E\right)\right\}$ 
where $\left(F_{1}, E\right),\left(F_{2}, E\right),\left(F_{3}, E\right),\left(F_{4}, E\right),\left(G_{1}, E\right),\left(G_{2}, E\right)$, $\left(G_{3}, E\right),\left(G_{4}, E\right),\left(G_{5}, E\right)$ are soft sets over $X$, defined as follows

$$
\begin{aligned}
& F_{1}\left(e_{1}\right)=\left\{h_{1}, h_{2}\right\} F_{1}\left(e_{2}\right)=\left\{h_{2}, h_{3}\right\} \\
& F_{2}\left(e_{1}\right)=\left\{h_{2}\right\} F_{2}\left(e_{2}\right)=\phi \\
& F_{3}\left(e_{1}\right)=\left\{h_{2}\right\} F_{3}\left(e_{2}\right)=\left\{h_{3}\right\} \\
& F_{4}\left(e_{1}\right)=\left\{h_{1}, h_{2}\right\} F_{4}\left(e_{2}\right)=\left\{h_{2}\right\} \\
& G_{1}\left(e_{1}\right)=\left\{h_{1}, h_{2}\right\} G_{1}\left(e_{2}\right)=\left\{h_{2}, h_{3}\right\} \\
& G_{2}\left(e_{1}\right)=\phi G_{2}\left(e_{2}\right)=\left\{h_{2}, h_{3}\right\} \\
& G_{3}\left(e_{1}\right)=\left\{h_{1}, h_{2}\right\} G_{3}\left(e_{2}\right)=\left\{h_{3}\right\} \\
& G_{4}\left(e_{1}\right)=X G_{4}\left(e_{2}\right)=\left\{h_{2}, h_{3}\right\} \\
& G_{5}\left(e_{1}\right)=\phi G_{5}\left(e_{2}\right)=\left\{h_{3}\right\}
\end{aligned}
$$

Then $\left(X, \tau_{1}, \tau_{2}, E\right)$ is a soft bitopological space. Now define

$\tau=\tau_{1} \cup \tau_{2}=\left\{\phi, X,\left(F_{1}, E\right),\left(F_{2}, E\right),\left(F_{3}, E\right),\left(F_{4}, E\right),\left(G_{1}, E\right)\right.$, $\left.\left(G_{2}, E\right),\left(G_{3}, E\right),\left(G_{4}, E\right),\left(G_{5}, E\right)\right\}$.

If take $\left(F_{2}, E\right) \cup\left(G_{2}, E\right)=(H, E)$, then

$H\left(e_{1}\right)=F_{2}\left(e_{1}\right) \cup G_{2}\left(e_{1}\right)=\left\{h_{2}\right\} \cup \phi=\left\{h_{2}\right\}$ and

$H\left(e_{2}\right)=F_{2}\left(e_{2}\right) \cup G_{2}\left(e_{2}\right)=\phi \cup\left\{h_{2}, h_{3}\right\}=\left\{h_{2}, h_{3}\right\}$

but $(H, E) \notin \tau$. Thus $\tau$ is not a soft topology on $X$.

\section{SEPARATION AXIOMS OF SOFT BITOPOLOGICAL SPACES}

3.1 Definition: In a soft bitopological space $\left(X, \tau_{1}, \tau_{2}, E\right)$

i) $\tau_{1}$ is said to be soft $T_{0}$ space w. r. t. $\tau_{2}$ if for each distinct points $x, y$ of $X$ then there exist a $\tau_{1}$ soft open set $(F, E)$ and a $\tau_{2}$ soft open set $(G, E)$ such that $x \in(F, E)$ and $y \notin(F, E)$ or $y \in$ $(G, E)$ and $x \notin(G, E)$.

Similarly $\tau_{2}$ is said to be soft $T_{0}$ space w. r. t. $\tau_{1}$ if for each distinct points $x, y$ of $X$ then there exist a $\tau_{2}$ soft open set $(F, E)$ and a $\tau_{1}$ soft open set $(G, E)$ such that $x \in(F, E)$ and $y \notin(F, E)$ or $y \in(G, E)$ and $x \notin(G, E)$.

$\left(X, \tau_{1}, \tau_{2}, E\right)$ is said to be pairwise soft $T_{0}$ space if $\tau_{1}$ is soft $T_{0}$ space w. r. t. $\tau_{2}$ and $\tau_{2}$ is soft $T_{0}$ space w. r. t. $\tau_{1}$.

ii) $\tau_{1}$ is said to be soft $T_{1}$ space w. r. t. $\tau_{2}$ if for each distinct points $x, y$ of $X$ then there exist a $\tau_{1}$ soft open set $(F, E)$ and a $\tau_{2}$ soft open set $(G, E)$ such that $x \in(F, E)$ and $y \notin(F, E)$ and $y \in$ $(G, E)$ and $x \notin(G, E)$.

Similarly $\tau_{2}$ is said to be soft $T_{1}$ space w. r. t. $\tau_{1}$ if for each distinct points $x, y$ of $X$ then there exist a $\tau_{2}$ soft open set $(F, E)$ and a $\tau_{1}$ soft open set $(G, E)$ such that $x \in(F, E)$ and $y \notin(F, E)$ and $y \in(G, E)$ and $x \notin(G, E)$.

$\left(X, \tau_{1}, \tau_{2}, E\right)$ is said to be pairwise soft $T_{1}$ space if $\tau_{1}$ is soft $T_{1}$ space w. r. t. $\tau_{2}$ and $\tau_{2}$ is soft $T_{1}$ space w. r. t. $\tau_{1}$.

iii) $\tau_{1}$ is said to be soft $T_{2}$ space w. r. t. $\tau_{2}$ if for each distinct points $x, y$ of $X$ then there exist a $\tau_{1}$ soft open set $(F, E)$ and a $\tau_{2}$ soft open set $(G, E)$ such that $x \in(F, E), y \in(G, E)$ and $(F, E) \cap(G, E)=\phi$.

Similarly $\tau_{2}$ is said to be soft $T_{2}$ space w. r. t. $\tau_{1}$ if for each distinct points $x, y$ of $X$ then there exist a $\tau_{2}$ soft open set $(F, E)$ and a $\tau_{1}$ soft open set $(G, E)$ such that $x \in(F, E), y \in(G, E)$ and $(F, E) \cap(G, E)=\phi$.

$\left(X, \tau_{1}, \tau_{2}, E\right)$ is said to be pairwise soft $T_{2}$ space if $\tau_{1}$ is soft $T_{2}$ space w. r. t. $\tau_{2}$ and $\tau_{2}$ is soft $T_{2}$ space w. r. t. $\tau_{1}$.

3.2 Thereom: Let $\left(X, \tau_{1}, \tau_{2}, E\right)$ be a soft bitopological space over $X$ and $Y$ be a non-empty subset of $X$. If $\left(X, \tau_{1}, \tau_{2}, E\right)$ is pairwise soft $T_{0}$ space then $\left(Y, \tau_{1 Y}, \tau_{2 Y}, E\right)$ is pairwise soft $T_{0}$ space.
Proof: Let $\left(X, \tau_{1}, \tau_{2}, E\right)$ be a soft bitopological space over $X$ and $x, y \in Y$ such that $x \neq y$. If $\left(X, \tau_{1}, \tau_{2}, E\right)$ is pairwise soft $T_{0}$ space, then there exist a $\tau_{1}$ soft open set $(F, E)$ and a $\tau_{2}$ soft open set $(G, E)$ such that $x \in(F, E)$ and $y \notin(F, E)$ or $y \in(G, E)$ and $x \notin(G, E)$. Now $x \in Y$ and $x \in(F, E)$. Hence $x \in Y \cap(F, E)=$ $\left({ }^{Y} F, E\right)$ where $(F, E) \in \tau_{1}$. Consider $y \notin(F, E)$ this means that $\alpha \in E$, then $y \notin Y \cap F(\alpha)$ for some $\alpha \in E$. Therefore $y \notin Y \cap(F, E)=\left({ }^{Y} F, E\right)$. Therefore $\tau_{1 Y}$ is soft $T_{0}$ space w. r. t. $\tau_{2 Y}$.

Similarly it can be prove that $\tau_{2 Y}$ is soft $T_{0}$ space w. r. t. $\tau_{1 Y}$, that is $y \in(G, E)$ and $x \notin(G, E)$ then $y \in\left({ }^{Y} G, E\right)$ and $x \notin\left({ }^{Y} G, E\right)$. Thus $\left(Y, \tau_{1 Y}, \tau_{2 Y}, E\right)$ is pairwise soft $T_{0}$ space.

3.3 Thereom: Let $\left(X, \tau_{1}, \tau_{2}, E\right)$ be a soft bitopological space over $X$ and $Y$ be a non-empty subset of $X$. If $\left(X, \tau_{1}, \tau_{2}, E\right)$ is pairwise soft $T_{1}$ space then $\left(Y, \tau_{1 Y}, \tau_{2 Y}, E\right)$ is pairwise soft $T_{1}$ space.

Proof: Let $\left(X, \tau_{1}, \tau_{2}, E\right)$ be a soft bitopological space over $X$ and $x, y \in Y$ such that $x \neq y$. If $\left(X, \tau_{1}, \tau_{2}, E\right)$ is pairwise soft $T_{1}$ space, then there exist a $\tau_{1}$ soft open set $(F, E)$ and a $\tau_{2}$ soft open set $(G, E)$ such that $x \in(F, E)$ and $y \notin(F, E)$ and $y \in(G, E)$ and $x \notin(G, E)$. Now $x \in Y$ and $x \in(F, E)$. Hence $x \in Y \cap$ $(F, E)=\left({ }^{Y} F, E\right)$ where $(F, E) \in \tau_{1}$. Consider $y \notin(F, E)$ this means that $\alpha \in E$, then $y \notin Y \cap F(\alpha)$ for some $\alpha \in E$. Therefore $y \notin Y \cap(F, E)=\left({ }^{Y} F, E\right)$. Now $y \in Y$ and $y \in(G, E)$. Hence $y \in Y \cap(G, E)=\left({ }^{Y} G, E\right)$ where $(G, E) \in \tau_{2}$. Consider $x \notin$ $(G, E)$ this means that $\alpha \in E$, then $x \notin Y \cap G(\alpha)$ for some $\alpha \in E$. Therefore $x \notin Y \cap(G, E)=\left({ }^{Y} G, E\right)$. Therefore $\tau_{1 Y}$ is soft $T_{1}$ space w. r. t. $\tau_{2 Y}$.

Similarly it can be prove that $\tau_{2 Y}$ is soft $T_{1}$ space w. r. t. $\tau_{1 Y}$, that is $y \in(G, E)$ and $x \notin(G, E)$ then $y \in\left({ }^{Y} G, E\right)$ and $x \notin\left({ }^{Y} G, E\right)$. Thus $\left(Y, \tau_{1 Y}, \tau_{2 Y}, E\right)$ is pairwise soft $T_{1}$ space.

3.4 Thereom: Let $\left(X, \tau_{1}, \tau_{2}, E\right)$ be a soft bitopological space over $X$. If $(x, E)$ is a soft closed set in $\tau_{2}$ for each $x \in X$ and $(y, E)$ is a soft closed set in $\tau_{1}$ for each $y \in X$ then $\left(X, \tau_{1}, \tau_{2}, E\right)$ is pairwise soft $T_{1}$ space.

Proof: Suppose that for each $x \in X,(x, E)$ is a soft closed set in $\tau_{2}$ then $(x, E)^{c}$ is soft open set in $\tau_{2}$. Let $x, y \in X$ such that $x \neq y$. For each $x \in X,(x, E)^{c}$ is a soft open set in $\tau_{2}$ such that $y \in(x, E)^{c}$ and $x \notin(x, E)^{c}$

Similarly for each $y \in X,(y, E)$ is a soft closed set in $\tau_{1}$ then $(y, E)^{c}$ is soft open set in $\tau_{1}$ such that $x \in(y, E)^{c}$ and $y \notin(y, E)^{c}$. Thus $\left(X, \tau_{1}, \tau_{2}, E\right)$ is pairwise soft $T_{1}$ space.

3.5 Thereom: Every pairwise soft $T_{1}$ space is pairwise soft $T_{0}$ space.

Proof: Let $\left(X, \tau_{1}, \tau_{2}, E\right)$ be a soft bitopological space over $X$ and $x, y \in X$ such that $x \neq y$. If $\left(X, \tau_{1}, \tau_{2}, E\right)$ is pairwise soft $T_{1}$ space. That is $\left(X, \tau_{1}, \tau_{2}, E\right)$ is pairwise soft $T_{1}$ space if $\tau_{1}$ is soft $T_{1}$ space w. r. t. $\tau_{2}$ and $\tau_{2}$ is soft $T_{1}$ space w. r. t. $\tau_{1}$. If $\tau_{1}$ is soft $T_{1}$ space w. r. t. $\tau_{2}$, then there exist a $\tau_{1}$ soft open set $(F, E)$ and a $\tau_{2}$ soft open set $(G, E)$ such that $x \in(F, E)$ and $y \notin(F, E)$ and $y \in(G, E)$ and $x \notin(G, E)$. Obviously $x \in(F, E)$ and $y \notin(F, E)$ or $y \in(G, E)$ and $x \notin(G, E)$. Therefore $\tau_{1}$ is soft $T_{0}$ space w. r. t. $\tau_{2}$

Similarly if $\tau_{2}$ is soft $T_{1}$ space w. r. t. $\tau_{1}$, then there exist a $\tau_{2}$ soft open set $(F, E)$ and a $\tau_{1}$ soft open set $(G, E)$ such that $x \in(F, E)$ and $y \notin(F, E)$ and $y \in(G, E)$ and $x \notin(G, E)$. Obviously $x \in$ $(F, E)$ and $y \notin(F, E)$ or $y \in(G, E)$ and $x \notin(G, E)$. Therefore $\tau_{2}$ is soft $T_{0}$ space w. r. t. $\tau_{1}$. Thus $\left(X, \tau_{1}, \tau_{2}, E\right)$ pairwise soft $T_{0}$ space. 
3.6 Remark: The converse of the Theorem 3.5 is not true.

3.7 Example: Let $X=\left\{h_{1}, h_{2}\right\}, E=\left\{e_{1}, e_{2}\right\}$ and $\tau_{1}=\{\phi, X,(F, E)\}$ and $\tau_{2}=\{\phi, X\}$, where $(F, E)$ is soft set over $X$, defined as follows

$F\left(e_{1}\right)=\phi F\left(e_{2}\right)=\left\{h_{1}\right\}$

Then $\left(X, \tau_{1}, \tau_{2}, E\right)$ is a soft bitopological space. Also $\left(X, \tau_{1}, \tau_{2}, E\right)$ is a pairwise soft $T_{0}$ space but which is not a pairwise soft $T_{1}$ space, because $h_{1}, h_{2} \in X$ with $h_{1} \neq h_{2}$ and there do not exist $\tau_{1}$ soft open set $\left(F_{1}, E\right)$ and $\tau_{2}$ soft open set $\left(G_{1}, E\right)$ such that $h_{1} \in\left(F_{1}, E\right)$ and $h_{2} \notin\left(F_{1}, E\right)$ and $h_{2} \in\left(G_{1}, E\right)$ and $h_{1} \notin\left(G_{1}, E\right)$.

3.8 Thereom: Let $\left(X, \tau_{1}, \tau_{2}, E\right)$ be a soft bitopological space over $X$ and $Y$ be a non-empty subset of $X$. If $\left(X, \tau_{1}, \tau_{2}, E\right)$ is pairwise soft $T_{2}$ space then $\left(Y, \tau_{1 Y}, \tau_{2 Y}, E\right)$ is pairwise soft $T_{2}$ space.

Proof: Let $\left(X, \tau_{1}, \tau_{2}, E\right)$ be a soft bitopological space over $X$ and $x, y \in Y$ such that $x \neq y$. If $\left(X, \tau_{1}, \tau_{2}, E\right)$ is pairwise soft $T_{2}$ space, then there exist a $\tau_{1}$ soft open set $(F, E)$ and a $\tau_{2}$ soft open set $(G, E)$ such that $x \in(F, E), y \in(G, E)$ and $(F, E) \cap(G, E)=\phi$. So for each $\alpha \in E, x \in F(\alpha), y \in G(\alpha)$ and $F(\alpha) \cap G(\alpha)=\phi$. This implies that $x \in Y \cap F(\alpha), y \in Y \cap G(\alpha)$ and $F(\alpha) \cap G(\alpha)=\phi$. Hence $x \in\left({ }^{Y} F, E\right), y \in\left({ }^{Y} G, E\right)$ and $\left({ }^{Y} F, E\right) \cap\left({ }^{Y} G, E\right)=\phi$, where $\left({ }^{Y} F, E\right)$ is soft open set in $\tau_{1 Y}$, and $\left({ }^{Y} G, E\right)$ is soft open set in $\tau_{2 Y}$. Therefore $\tau_{1 Y}$ is soft $T_{2}$ space w. r. t. $\tau_{2 Y}$

Similarly it can be prove that $\tau_{2 Y}$ is soft $T_{2}$ space w. r. t. $\tau_{1 Y}$. Thus $\left(Y, \tau_{1 Y}, \tau_{2 Y}, E\right)$ is pairwise soft $T_{2}$ space.

3.9 Thereom: Every pairwise soft $T_{2}$ space is pairwise soft $T_{1}$ space.

Proof: Let $\left(X, \tau_{1}, \tau_{2}, E\right)$ be a soft bitopological space over $X$ and $x, y \in X$ such that $x \neq y$. If $\left(X, \tau_{1}, \tau_{2}, E\right)$ is pairwise soft $T_{2}$ space. That is $\left(X, \tau_{1}, \tau_{2}, E\right)$ is pairwise soft $T_{2}$ space if $\tau_{1}$ is soft $T_{2}$ space w. r. t. $\tau_{2}$ and $\tau_{2}$ is soft $T_{2}$ space w. r. t. $\tau_{1}$. If $\tau_{1}$ is soft $T_{2}$ space w. r. t. $\tau_{2}$, then there exist a $\tau_{1}$ soft open set $(F, E)$ and a $\tau_{2}$ soft open set $(G, E)$ such that $x \in(F, E), y \in(G, E)$ and $(F, E) \cap(G, E)=\phi$. Obviously $x \in(F, E)$ and $y \notin(F, E)$ and $y \in(G, E)$ and $x \notin(G, E)$. Therefore $\tau_{1}$ is soft $T_{1}$ space w. r. t. $\tau_{2}$

Similarly if $\tau_{2}$ is soft $T_{2}$ space w. r. t. $\tau_{1}$, then there exist a $\tau_{2^{-}}$ soft open set $(F, E)$ and a $\tau_{1}$-soft open set $(G, E)$ such that $x \in$ $(F, E), y \in(G, E)$ and $(F, E) \cap(G, E)=\phi$. Obviously $x \in$ $(F, E)$ and $y \notin(F, E)$ and $y \in(G, E)$ and $x \notin(G, E)$. Therefore $\tau_{2}$ is soft $T_{1}$ space w. r. t. $\tau_{1}$. Thus $\left(X, \tau_{1}, \tau_{2}, E\right)$ pairwise soft $T_{1}$ space.

Acknowledgment: The author would like to thank the reviewers for their valuable comments and helpful suggestions for improvement of the original manuscript.

\section{REFERENCES}

[1] N. Cagman, S. Karatas and S. Enginoglu, Soft topology, Comput. Math. Appl. 62 (2011), 351-358.

[2] D. Chen, The parametrization reduction of soft sets and its applications, Comput. Math. Appl. 49 (2005) 757-763.

[3] F. Feng, Y. B. Jun, X. Z. Zhao, Soft semirings, Comput. Math. Appl. 57 (2009), 1547-1553.
[4] J.C.Kelly, Bitopological spaces, Proc. London Math. Soc., 13 (1963), 71-81.

[5] P. K. Maji, R. Biswas, R. Roy, Soft set theory, Comput. Math. Appl. 45 (2003) 555-562.

[6] D.A. Molodtsov, Soft set theory-first results, Comput. Math. Appl. 37 (1999) 19-31.

[7] Muhammad Shabir, Munazza Naz, On soft topological spaces, Comput. Math. Appl., Vol. 61, 2011, pp. 1786-1799.

[8] C.W.Patty, Bitopological spaces, Duke Math. J., 34 (1967), $387-$ 392.

[9] I.LReilly, On bitopological separation properties, Nanta Math., 29(1972), 14-25. 